\title{
Polarimetric investigation of Uranus and Neptune
}

\author{
F. Joos and H. M. Schmid \\ Institute of Astronomy, ETH Zurich, CH-8092 Zurich, Switzerland \\ email: fjoos@astro.phys.ethz.ch
}

\begin{abstract}
We present ground-based disc resolved polarimetric imaging and spectropolarimetry of Uranus and Neptune in the wavelength range from 530 to $930 \mathrm{~nm}$ with the goal to constrain the expected polarization of extra-solar planets with similar atmospheric properties. We find for both planets a high limb polarization as expected for atmospheres with significant Rayleigh scattering layers. The limb polarization is enhanced in the strong methane absorption bands when compared to the adjacent continuum. From the observed polarization features we conclude that extra-solar planets with atmospheric properties similar to Uranus and Neptune are strongly polarized and well suited for polarimetric detection and investigation.
\end{abstract}

Keywords. Polarimetry, exoplanets, Uranus, Neptune.

\section{Introduction}

Reflected light from planets is polarized due to scattering. This basic property provides the possibility of detecting and characterizing extra-solar planets with polarimetry. However, it is hard to predict the amount and the wavelength dependency of polarization present in a "typical" extra-solar planet. Currently, observational results on the polarization of planets are only available for solar system targets. Therefore we investigate the scattering polarization of solar system planets in order to extrapolate their polarimetric properties to the case of extra-solar planets.

A typical extra-solar planet is expected to be observed for a phase angle $\alpha \approx 60^{\circ}$ to $120^{\circ}$ (Fig. 1). Observations of our solar system planets for similar $\alpha$ 's are desirable, but from Earth all outer planets can only be observed for small phase angles (back-scattering). For Jupiter and Saturn there exist polarimetric observations for $\alpha \approx 90^{\circ}$ from the Pioneer and Galileo spacecrafts (e.g. Smith \& Tomasko 1984; Tomasko \& Dose 1984; Braak et al. 2002). These data can directly be used as proxy for extra-solar planets. For Jupiter, for example, the polarization for $\alpha \approx 90^{\circ}$ is up to $50 \%$ at the poles and only $5 \%$ near the equator (R-band). Polarimetric measurements for small phase angles, $\alpha \leqslant 12^{\circ}$ taken from the ground show a polarization of about $7 \%$ at the poles of Jupiter and only $0.5 \%$ at the equator (e.g. Hall \& Riley 1976; Joos et al. 2005). The polarization for Jupiter is much higher at the poles because they are covered with a Rayleigh scattering layer. At the equator, the radiation is predominantly reflected by clouds and the resulting polarization is small.

For Uranus and Neptune no similar space observations could be found in the literature. For this reason we have taken disc resolved imaging polarimetry and longslit spectropolarimetry of Uranus and Neptune to investigate their polarimetric properties based on the limb-polarization effect.

For disc resolved observations there exists a well known second-order scattering effect in Rayleigh atmospheres, which produces an enhanced polarization at the limbs in a 
backscattering situation, see van de Hulst 1980. This effect is also found for the first time with our ground based observations of Uranus and Neptune. The measured limb polarization combined with analytical models allows to estimate roughly the amount of polarization of Uranus and Neptune for phase angles around $90^{\circ}$.

Figure 1. Schematic illustration of the expected scattering polarization (Rayleigh scattering) from an extra-solar planet in a low (left) and high (right) inclination system. The parent star is indicated by an asterisk. For small apparent separations (dotted circle) the planet is too close to the star to be detectable. The bars indicate strength and direction of polarization which is particularly high for
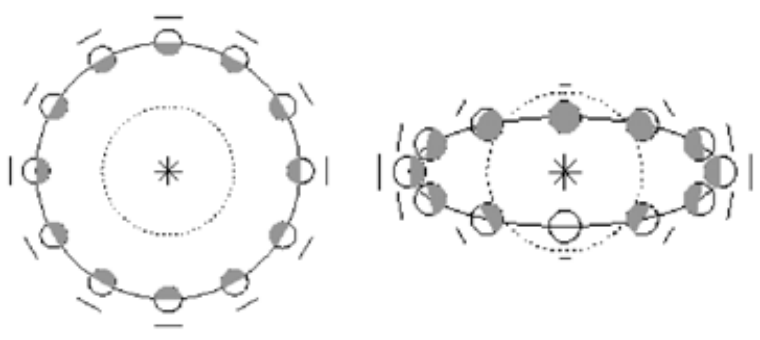
scattering angles near $90^{\circ}$.

\section{Observations \& results}

Our polarimetric observations were taken in Nov. 2003 with EFOSC2 attached at the Cassegrain focus of the ESO $3.6 \mathrm{~m}$ telescope. EFOSC2 is a multi-mode imager and grism spectrograph equipped with a Wollaston prism and a rotatable superachromatic halfwave plate for linear polarimetry and spectropolarimetry. The employed grism provides a coverage from about 5200 to $9350 \AA$ and a resolution of $12.8 \AA$ for a 1 " wide slit. The slit was 20" long and 0.5 " (Uranus) or 1.5" (Neptune) wide and was placed in NorthSouth direction through the middle of the disc. The pixel scale was 0.157 " / pixel and the seeing was better than 1". The diameter of Uranus was 3.5" and for Neptune 2.2". The intensity spectra have been calibrated with the albedo spectrum from Karkoschka 1994. We have binned our spectral data into $30 \AA$ bins in order to reduce the noise. In addition we have taken imaging polarimetry in several filters.

In the spectropolarimetric plots we show data for the limb (average of northern and southern limb), the central region and the total slit of Uranus and Neptune.

For Uranus we detect a peak polarization up to $3 \%$ at the limb and about $1 \%$ for the entire slit. We also show a cut through the planetary disc in a methane absorption band to point out the spatial features (Fig. 2).

Neptune has a diameter of 2.2", and our spectropolarimetric data contain almost the entire planet except some parts of the eastern and western limbs. We detected for Neptune a peak polarization of about $1 \%$ in the methane absorption bands (Fig. 4 left). For both planets we show imaging polarimetry of the Stokes Q flux in the i-band at $0.8 \mu \mathrm{m}$ (Fig. 3 for Uranus and Fig. 4 right for Neptune).

The limb polarization is for both planets clearly visible in the spectropolarimetric data as well as in the imaging polarimetry, whereas at the disc centers the polarization is close to zero. The imaging polarimetry shows that the polarization is enhanced along the entire limb of Uranus and Neptune and not only at the poles as for Jupiter. Further we find enhanced polarization in the methane absorption bands compared to the adjacent continuum. We also see an enhanced continuum polarization towards the blue. 

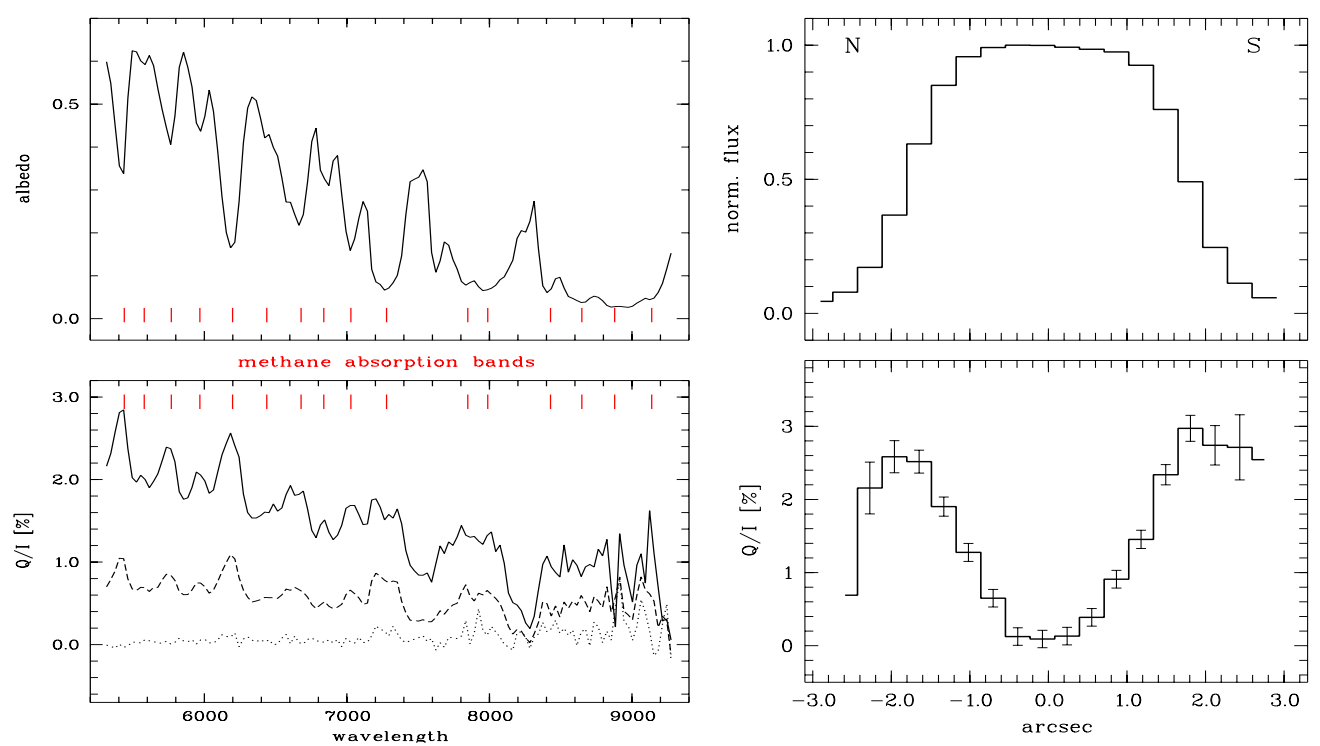

Figure 2. Spatially resolved spectropolarimetry of Uranus:

left: The albedo spectrum in the upper and the Stokes Q/I spectra in the lower panel where the solid line shows the limb position, the dashed line the total slit and the dotted line the central part of the planet. The vertical marks indicate the wavelengths of the methane absorptions.

right: Cut through the disk from North $(\mathrm{N})$ to South $(\mathrm{S})$ in the methane band at $6190 \AA$ $( \pm 10 \AA)$, normalized flux (upper panel) and Stokes Q/I with error bars (lower panel) versus the radial position on the planet.

Figure 3. Uranus: Stokes Q image $\left(\mathrm{I}_{0}-\mathrm{I}_{90}\right)$ in the i-band, white is positive and indicates a vertical polarization (North-South). North is up and East it left. The greyscale is normalized to the peak intensity and ranges from $-0.5 \%$ (black) to $+0.5 \%$ (white). The planetary South pole, the equator and the geometrical limb are marked.

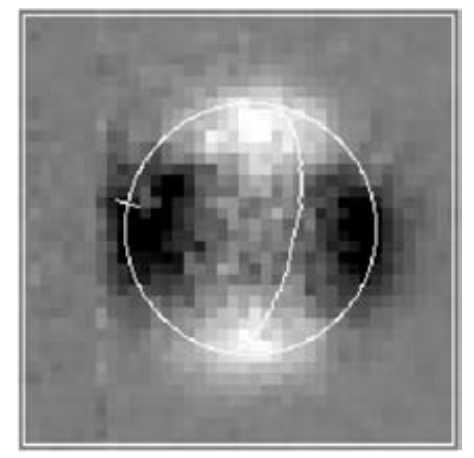

\section{Discussion and Conclusions}

Our polarimetric measurements of Uranus and Neptune show:

- a high limb polarization is present in both planets along the entire limb. This indicates a high scattering polarization $(\gtrsim 20 \%)$ for phase angles near $90^{\circ}$ (from comparison with observations of Jupiter's poles and analytic models). Thus extra-solar planets with similar properties as Uranus and Neptune are detectable with polarimetry.

- enhanced polarization in the methane absorption bands compared to the adjacent continuum. A higher polarization in the methane bands seems to be a generic effect for reflected light from planetary atmospheres. This is due to the high absorption in the methane bands which reduces predominantly the multiple scattered light and the 

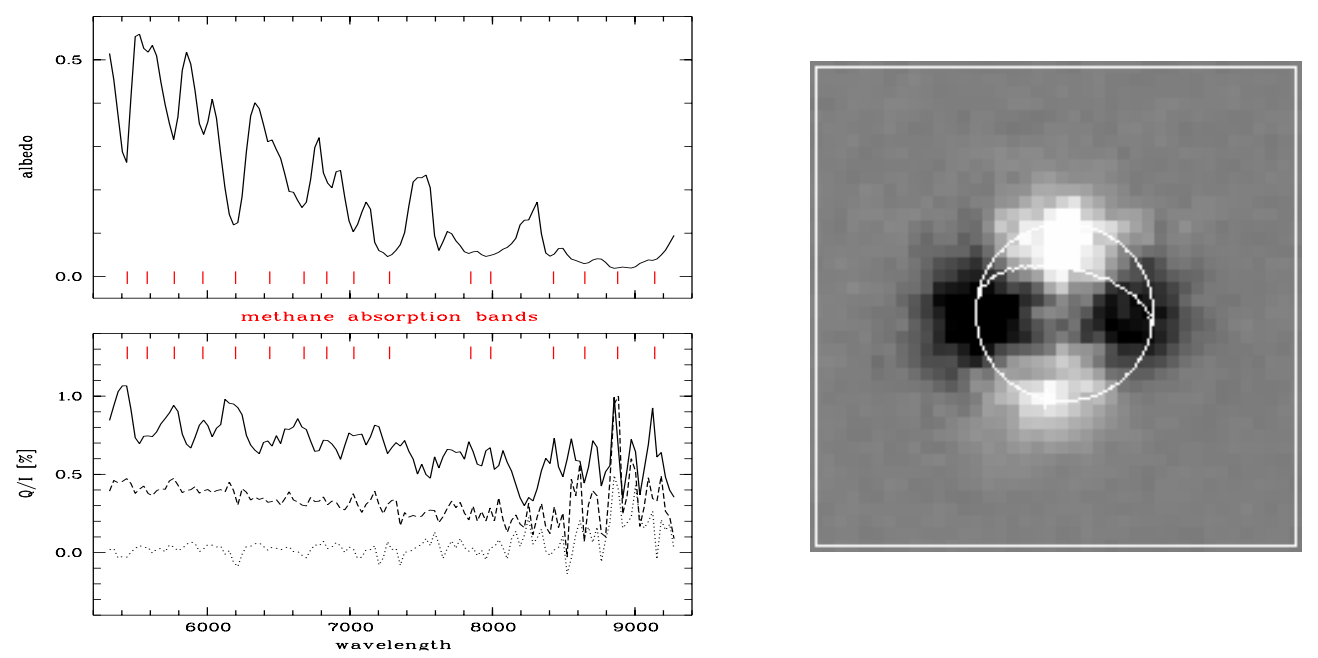

Figure 4. Spectropolarimetry (left) and imaging polarimetry (right) of Neptune. Details are the same as in Fig. (2) and in Fig. (3), respectively but for Neptune.

deeply penetrating photons reflected from cloud layers. Photons reflected in the highest atmospheric layers after one or two scatterings (the latter produce the limb polarization), are much less affected by absorption. This enhancement in the methane bands provides a tool for the verification of a planet detection.

- The enhanced continuum polarization towards the blue is typical for a Rayleigh scattering atmosphere. Since Rayleigh scattering is much stronger for short wavelengths, blue photons will penetrate less deep into the atmosphere and scatter at the high lying polarizing layers, whereas the deeply penetrating red photons have a higher probability to scatter on weakly polarizing clouds.

Based on observations of solar system planets we conclude that Uranus and Neptunelike extra-solar planets will show substantial polarization. The polarization signal is low for planets with high clouds in the atmosphere (e.g. equatorial region of Jupiter). A high polarization, as seen in Uranus and Neptune indicates that there exists an optically thick Rayleigh scattering layer above the clouds. The polarization in molecular bands like methane yield additional information about the composition and structure of the atmospheres. Thus polarimetry provides a powerful tool for the future investigation of extra-solar planets.

\section{References}

Braak, C.J., de Haan, J.F., Hovenier, J.W., \& Travis, L.D. 2002, Icarus 157, 401

Hall, J.S. \& Riley, L.A. 1976, Icarus 29, 231

Joos, F., Schmid, H.M., Gisler, D., Feldt, M., Brandner, W., Stam, D., Quirrenbach, A., \& Stuik, R. 2005, in: Adamson, A., Aspin, C., Davis, C.J., Fujiyoshi, T. (eds.), ASP Conf. Ser. (in press)

Karkoschka, E. 1994, Icarus 111, 174

Schmid, H.M., Joos, F., \& Tschan, D. 2005, A\&\&A (submitted)

Smith, P.H. \& Tomasko, M.G. 1984, Icarus 58, 35

Tomasko, M.G. \& Doose, L.R. 1984, Icarus 58, 1

van de Hulst, H.C. 1980, Multiple Light Scattering, 2nd Volume (Academic Press) 